\title{
How Oxygen Affects the CMDs and Predicted Ages of Extreme Population II Stars
}

\author{
Don A. VandenBerg \\ University of Victoria, Dept. of Physics \& Astronomy, Box 3055, \\ Victoria, B.C. V8W $3 P 6$ Canada \\ R.A. Bell \\ University of Maryland, Dept. of Astronomy, College Park, \\ $M D$ 20742-2421 U.S.A.
}

\begin{abstract}
Stellar evolutionary models for $[\mathrm{Fe} / \mathrm{H}]=-2.27$ have been constructed to examine how predicted C-M diagrams, age versus turnoffluminosity relations, the luminosities of the red-giant "bump" and tip, and the $T_{\text {eff }} /$ color of a zero-age horizontal branch model of fixed mass are affected by varying the assumed abundance of oxygen (with and without enhancements in the other $\alpha$-elements).
\end{abstract}

\section{Introduction}

The consequences of varying the assumed oxygen abundance on the turnoff luminosities of metal-poor stars have been evaluated by Simoda \& Iben (1970), Rood \& Crocker (1985), and VandenBerg (1985): at a fixed $L_{\mathrm{TO}}$, ages are decreased by $\approx 3$ Gyr if the $\mathrm{O} / \mathrm{Fe}$ number abundance ratio is increased by a factor of 10 over the solar value. Rood \& Crocker have also shown that the luminosity of the Helium Flash and of the red-giant "bump" (the local enhancement in the number of giants that arises when the H-burning shell contacts the chemical abundance discontinuity left behind when the convective envelope reached its deepest penetration) are fairly sensitive functions of the adopted $[\mathrm{O} / \mathrm{Fe}]$ value. Moreover, their work has demonstrated that the predicted distribution of stars on the horizontal branch depends very strongly on the assumed $\mathrm{O}$ abundance.

The main purpose of this study is to update previous work in the light of the many improvements that have been made to our understanding of the basic physics of stars. Of particular interest are models for low iron abundances given that canonical estimates of $[\mathrm{O} / \mathrm{Fe}]$ near 0.3 (e.g., the reviews by Kraft 1994, McWilliam 1997) have been called into question by Israelian et al. (1998) and Boesgaard et al. (1999), who have obtained $[\mathrm{O} / \mathrm{Fe}] \gtrsim 1$ at $[\mathrm{Fe} / \mathrm{H}] \lesssim-2$. To be specific, we have constructed stellar models for $[\mathrm{Fe} / \mathrm{H}]=-2.27$ and four different heavy-element mixtures: (i) $[\mathrm{m} / \mathrm{H}]=0.0$, (ii) $[\mathrm{O} / \mathrm{Fe}]=0.3$, all other metals in scaled-solar proportions, (iii) $[\alpha / \mathrm{Fe}]=0.3$, and (iv) $[\alpha / \mathrm{Fe}]=0.3$, but $[\mathrm{O} / \mathrm{Fe}]=1.0$. Model atmospheres for each of these choices have been computed to provide fully-consistent boundary conditions and synthetic colors. 


\section{The Results}

At the $[\mathrm{Fe} / \mathrm{H}]$ value that we have considered, nucleosynthesis and opacity effects are approximately equally responsible for the reduction in $L_{\mathrm{TO}}$ at a fixed age when the $[\mathrm{O} / \mathrm{Fe}]$ ratio is increased (see VandenBerg et al. 2000). Furthermore, in excellent agreement with previous work, isochrones based on the latest nuclear reaction rates, opacities, and equation of state predict about a 1 Gyr reduction in age with each 0.3 dex increase in $[\mathrm{O} / \mathrm{Fe}]$. This is illustrated in Table 1, which shows that the age inferred from scaled-solar-mix models is about $1 \mathrm{Gyr}$ $(6.6 \%)$ less than that derived from $[\alpha / \mathrm{Fe}]=0.3$ computatations, whereas a smaller age by just over $2 \mathrm{Gyr}(14.5 \%)$ is found if $[\mathrm{O} / \mathrm{Fe}]=1.0$. Note that ages derived from isochrones for $[\mathrm{O} / \mathrm{Fe}]=0.3$ are very similar to those obtained from $[\alpha / \mathrm{Fe}]=0.3$ computations, which demonstrates the overwhelming importance of oxygen among the $\alpha$-elements.

Table 1. Predicted Age at a Turnoff Luminosity Corresponding to $\log L / L_{\odot}=0.373$.

\begin{tabular}{ccclcc}
\hline Mix & {$[\mathrm{Fe} / \mathrm{H}]$} & $Y$ & Heavy-Element Mixture & $\mathrm{t}(\mathrm{Gyr})$ & $\delta(\%)$ \\
\hline $\mathrm{A}$ & -2.27 & 0.2352 & {$[\mathrm{~m} / \mathrm{Fe}]=0.0$} & 14.92 & +6.6 \\
$\mathrm{~B}$ & -2.27 & 0.2352 & {$[\mathrm{~m} / \mathrm{Fe}]=0.0,[\mathrm{O} / \mathrm{Fe}]=0.3$} & 14.12 & +0.9 \\
$\mathrm{C}$ & -2.27 & 0.2352 & {$[\alpha / \mathrm{Fe}]=0.3$} & 14.00 & - \\
$\mathrm{D}$ & -2.27 & 0.2352 & {$[\alpha / \mathrm{Fe}]=0.3,[\mathrm{O} / \mathrm{Fe}]=1.0$} & 11.97 & -14.5 \\
\hline
\end{tabular}

Grundahl et al. (2000) have recently shown that M92 must have a distance modulus close to $(m-M)_{V}=14.64$ if the cluster subgiants are fitted to HD 140283, which has $\sigma_{\pi} / \pi=0.06$ (from Hipparcos), a metallicity that is within 0.25 dex of that of M 92, and a comparable reddening. The inferred age at this distance is close to $15.5 \mathrm{Gyr}$ if models for $[\alpha / \mathrm{Fe}]=0.3$ are assumed, or 13.2 Gyr if $[\alpha / \mathrm{Fe}]=0.3$ and $[\mathrm{O} / \mathrm{Fe}]=1.0$ is more realistic (see the plots provided by VandenBerg \& Bell 2001). The importance of having accurate $\mathrm{O}$ abundances for abolute ages and age-metallicity relations is obvious.

Table 2 lists some of the properties of $0.8 \mathcal{M}_{\odot}$ giants having the chemical abundance mixtures considered in this study. As the $[\mathrm{O} / \mathrm{Fe}]$ value increases, $M_{V}^{\text {bump }}$ becomes fainter, $M_{V}^{\text {tip }}$ becomes brighter, and the helium core mass at the Helium Flash $\left(\mathcal{M}_{c}\right)$ decreases. As M 92's bump occurs at $V=14.65 \pm 0.05$ (Ferraro et al. 1999), $M_{V}^{\text {bump }} \approx 0.0$ if the distance modulus based on HD 140283 is assumed. This agrees well with the predictions of the models for mix " $D$ ". However, if some amount of inward overshooting of the convective envelope occurs in real stars, then the models for the other heavy-element mixtures may be preferable.

Reduced core masses and increased $\mathrm{O}$ abundances imply fainter and redder horizontal branches, as illustrated in Figure 1. It is also clear that the masses of stars within the instability strip must increase dramatically with increasing $[\mathrm{O} / \mathrm{Fe}]$; and thus, cluster-to-cluster variations in the abundance of oxygen may be at least partly responsible for the " $2^{\text {nd }}$ parameter" and Oosterhoff phenomena exhibited by the Galaxy's globular clusters. 
Table 2. RGB Bump and RGB Tip Properties of a $0.8 \mathcal{M}_{\odot}$ Star Having $[\mathrm{Fe} / \mathrm{H}]=-2.27$ and Different Heavy-Element Mixtures

\begin{tabular}{cllllll}
\hline Mix & $\log T_{\text {eff }}^{\text {bump }}$ & $M_{V}^{\text {bump }}$ & $t^{\text {tip }}$ & $\mathcal{M}_{c}^{\text {tip }}$ & $\log T_{\text {eff }}^{\text {tip }}$ & $M_{V}^{\text {tip }}$ \\
\hline A & 3.6881 & -0.46 & 13.43 & 0.5014 & 3.6373 & -2.73 \\
B & 3.6898 & -0.35 & 13.43 & 0.4984 & 3.6362 & -2.75 \\
C & 3.6875 & -0.33 & 13.51 & 0.4985 & 3.6311 & -2.71 \\
D & 3.6925 & -0.02 & 13.65 & 0.4901 & 3.6278 & -2.77 \\
\hline \hline
\end{tabular}

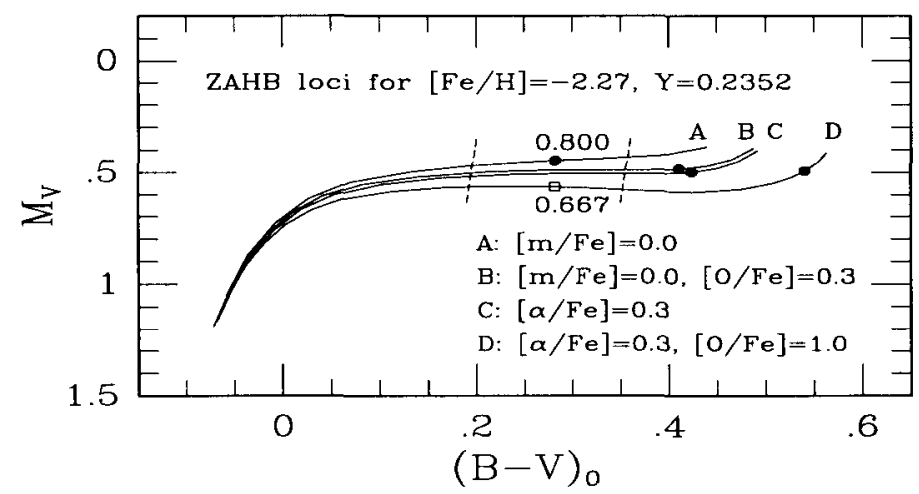

Figure 1. ZAHB loci for the 4 element mixtures considered. Filled circles indicate the locations of $0.8 \mathcal{M}_{\odot}$ models in each case.

(For a much expanded version of this paper, reference should be made to VandenBerg \& Bell 2001.)

\section{References}

Boesgaard, A.M., King, J.R., Deliyannis, C.P., \& Vogt, S.S. 1999, AJ, 117, 492 Ferraro, F.R., Messineo, M., Fusi Pecci, F., et al. 1999, AJ, 118, 1738 Grundahl, F., VandenBerg, D.A., Bell, R.A., et al. 2000, AJ, in press Israelian, G., García López, R.J., \& Rebolo, R. 1998, ApJ, 507, 805 Kraft, R.P. 1994, PASP, 106, 553

McWilliam, A. 1997, ARA\&A, 35, 503

Rood, R.T., \& Crocker, D.A. 1985, in Production and Distribution of C, N, O Elements, eds. I.J. Danziger, F. Matteucci, \& K. Kjär (Garching bei München: ESO), 61

Simoda, M., \& Iben, I., Jr. 1970, ApJS, 22, 81

VandenBerg, D.A. 1985, in Production and Distribution of C, N, O Elements, eds. I.J. Danziger, F. Matteucci, \& K. Kjär (Garching bei München), 73

VandenBerg, D.A., \& Bell, R.A. 2001, New Astron. Rev., in press

VandenBerg, D.A., Swenson, F.J., Rogers, F.J., et al. 2000, ApJ, 532, 430 